OPEN ACCESS

Edited by:

Weiyang Lou,

Zhejiang University, China

Reviewed by:

Junjie Zhang,

Fuyang City People's Hospital, China

Huilin Zheng,

Zhejiang University of Science and

Technology, China

*Correspondence:

Jing Chen

b1818220@zju.edu.cn

Specialty section:

This article was submitted to Epigenomics and Epigenetics,

a section of the journal

Frontiers in Cell and Developmental

Biology

Received: 20 December 2021 Accepted: 06 January 2022

Published: 25 January 2022

Citation:

Liu J, Xiao S and Chen J (2022) Development of an Inflammation-

Related IncRNA-miRNA-mRNA

Network Based on Competing Endogenous RNA in Breast Cancer at

Single-Cell Resolution.

Front. Cell Dev. Biol. 10:839876.

doi: 10.3389/fcell.2022.839876

\section{Development of an} Inflammation-Related IncRNA-miRNA-mRNA Network Based on Competing Endogenous RNA in Breast Cancer at Single-Cell Resolution

\author{
Jingxing Liu ${ }^{1}$, Shuyuan $\mathrm{Xiao}^{2}$ and Jing Chen ${ }^{3 *}$ \\ ${ }^{1}$ Department of Intensive Care Unit, Changxing People's Hospital of Zhejiang, Huzhou, China, ${ }^{2}$ Department of Anesthesiology, \\ The First Affiliated Hospital, College of Medicine, Zhejiang University, Hangzhou, China, ${ }^{3}$ Department of Oncology, The First \\ Affiliated Hospital of Jiaxing University, Jiaxing, China
}

The role and mechanism of inflammation in breast cancer is unclear. This study aims to probe the relationship between inflammation and long non-coding RNAs (IncRNAs) and to stablish an inflammation-related competing endogenous RNA (ceRNA) network in breast cancer. Inflammation-related IncRNAs and target genes were screened based on the data from four single-cell RNA sequencing (scRNA-seq) studies and miRNAs were bioinformatically predicted according to ceRNA hypothesis. A series of in silico analyses were performed to construct an inflammation-related ceRNA network in breast cancer. Consequently, a total of seven inflammation-related IncRNAs were selected, after which LRRC75A-AS1 was identified as the most potential IncRNA in view of its expression and prognostic predictive value in breast cancer. Finally, an inflammation-related ceRNA network in breast cancer at the single cell level was established based on IncRNA LRRC75A-AS1, miR-3127-5p, miR-2114-3p, RPL36 and RPL27A mRNAs. Collectively, the IncRNA LRRC75A-AS1 and the LRRC75A-AS1based on ceRNA network may exert crucial roles in modulating inflammation response during the initiation and progression of breast cancer.

Keywords: inflammation, breast cancer, LRRC75A-AS1, microRNA (miRNA), prognosis, single cell RNA sequencing (scRNA-seq)

\section{INTRODUCTION}

Breast cancer ranks the most common type of human cancer and is also the leading cause of cancerrelated deaths in women all over the world (Sung et al., 2021). The prognosis of patients with breast cancer, especially triple-negative breast cancer and HER2-positive breast cancer, is still unsatisfactory, in spite of huge advancements in the aspect of its diagnosis and therapy have been achieved during the past decades (de la Mare et al., 2014). More work and efforts need to be put in the extensive and in-depth research regarding the molecular mechanism of breast carcinogenesis and progression.

When Rudolph Virchow noted leucocytes in tumor tissues in 1863 and proposed the theory of "lymphoreticular infiltrate", the origin of cancer at sites of inflammation came into researchers' sight 
TABLE 1 | The potential IncRNAs significantly associated with inflammation in breast cancer.

\begin{tabular}{lccc}
\hline \multirow{2}{*}{ LncRNA } & \multicolumn{3}{c}{ Correlation efficient } \\
\cline { 2 - 4 } & EXP0052 & EXP0053 & EXP0054 \\
\hline LRRC75A-AS1 & -0.311 & -0.340 & - \\
RGPD4-AS1 & -0.313 & -0.321 & - \\
AC010255.1 & -0.464 & - & -0.548 \\
MALAT1 & -0.469 & -0.321 & - \\
AC159540.2 & -0.369 & - & -0.349 \\
AC091891.2 & -0.539 & - & -0.422 \\
FAM239A & - & -0.318 & -0.356
\end{tabular}

(Balkwill and Mantovani, 2001). After that inflammation has been recognized to contribute to malignant transformation of several types of human malignancies, including hepatocellular carcinoma, colorectal cancer and gastric cancer (Multhoff et al., 2011). Inflammation is also a key component of breast carcinogenesis (Danforth, 2021). However, its roles and molecular mechanism in the initiation and progression of breast cancer is still unclear and need to be further elucidated.

Single-cell RNA sequencing (scRNA-seq) technology, a powerful tool to investigate cell heterogeneity, bringing new strategies to deeply understand the characteristics and behaviors of human cancer (including breast cancer) at the single-cell level (Ren et al., 2021). In this study, we first screened out several inflammation-related lncRNAs in breast cancer using four breast cancer-related scRNA-seq studies, then predicted and analyzed corresponding downstream microRNAs (miRNAs) based on the competing endogenous RNA (ceRNA) hypothesis (Salmena et al., 2011), and finally also identified inflammation-related target gens in breast cancer at the single-cell level. At the end, an inflammation-related lncRNA-miRNA-mRNA triple RNA regulatory network in breast cancer has been established.

\section{RESULTS}

\section{Identification of LRRC75A-AS1 as a Potential Inflammation-Related Long Non-Coding RNA in Breast Cancer}

ScRNA-seq provides an unprecedented opportunity to probe the functional heterogeneity of cancer cells. It has been widely acknowledged that inflammation is closely linked to occurrence and progression of various types of human cancers, including breast cancer. To elucidate the underlying molecular mechanism of the inflammation in breast cancer, four breast cancer-associated scRNA-seq studies were collected (Balavenkatraman et al., 2011; Braune et al., 2016; Jordan et al., 2016; Chung et al., 2017). Consequently, a total of 656 lncRNAs that were significantly associated with inflammation in breast cancer were screened out (Data not shown). Among these lncRNAs, only seven members, consisting of LRRC75A-AS1, RGPD4-AS1, AC010255.1, MALAT1, AC159540.2, AC091891.2 and
FAM239A, appear in more than one scRNA-seq studies. As listed in Table 1, all the seven lncRNAs were negatively correlated with inflammation in breast cancer. Next, we successively determined the expression levels of the seven lncRNAs in breast cancer by GEPIA database and starBase database. As presented in Figure 1, only LRRC75A-AS1 expression was markedly decreased in breast cancer when compared with normal controls in both two databases. These findings suggested that LRRC75A-AS1 might be the most potential inflammation-related lncRNA in breast cancer.

\section{Survival Analysis for LRRC75A-AS1 in Various Immunocyte-Enriched/Decreased Breast Cancer}

As mentioned above, LRRC75A-AS1 was identified as a potential inflammation-related lncRNA in breast cancer. As is known to all, inflammation and immunity are two ends of the balance in human body. Thus, we performed survival analysis for inflammation-related LRRC75A-AS1 in breast cancer enriched or decreased with various immunocytes. Nine different immunocytes, involving basophil, B cell, $\mathrm{CD}^{+} \mathrm{T}$ cell, $\mathrm{CD}^{+} \mathrm{T}$ cell, eosinophil, macrophage, mesenchymal stem cell, natural killer $\mathrm{T}$ cell and regulatory $\mathrm{T}$ cell, were included for this survival analysis. As shown in Figure 2, high expression of LRRC75A-AS1 indicated favorable overall survival in breast cancer enriched with basophil or natural killer T cell. Moreover, upregulation of LRRC75A-AS1 was positively correlated with good overall survival in breast cancer decreased with B cell, $\mathrm{CD}^{+} \mathrm{T}$ cell, eosinophil or mesenchymal stem cell (Figure 3). No significant prognostic values of LRRC75A-AS1 in breast cancer enriched or decreased with other immunocytes were observed. Taken together, some immunocytes, including basophil, natural killer $\mathrm{T}$ cell, B cell, $\mathrm{CD}^{+} \mathrm{T}$ cell, eosinophil or mesenchymal stem cell, might be involved in interaction with LRRC75A-AS1mediated inflammation in breast cancer.

\section{Prediction and Analysis of Downstream Potential miRNAs of LRRC75A-AS1 in Breast Cancer}

To further explore the downstream action mechanism of inflammation-related LRRC75A-AS1 in breast cancer, miRNet was employed to predict the miRNAs that potentially bind to LRRC75A-AS1. A total of 70 miRNAs were identified. For better visualization, a LRRC75A-AS1miRNA network was established using Cytoscape software (Figure 4). According to competing endogenous RNA (ceRNA) hypothesis, these should be negative expression correlation between IncRNA and miRNA. Therefore, expression correlation of LRRC75A-AS1 with its predicted miRNAs in breast cancer was calculated using TCGA breast cancer data. As listed in Table 2, 15 of 70 miRNAs were significantly inversely associated with LRRC75A-AS1 in breast cancer. Then, we further analyzed the expression levels of the 15 negatively correlated miRNAs in breast 


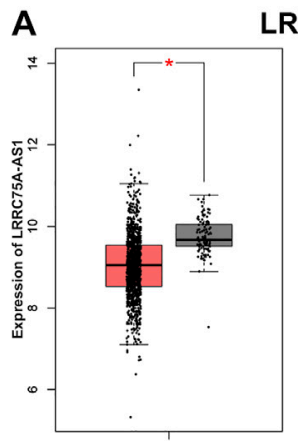

BRCA
$(\operatorname{num}(\mathrm{T})=1085 ; \operatorname{num}(\mathrm{N})=112)$

C

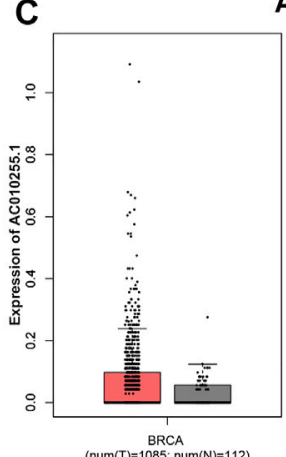

AC010255.1

$\mathbf{E}$

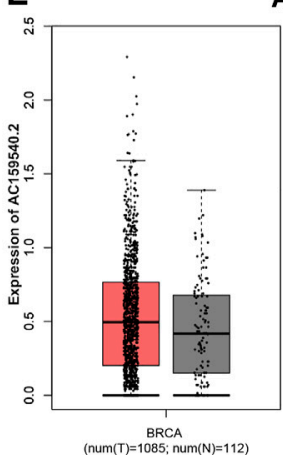

G

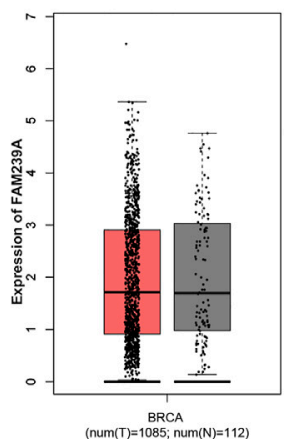

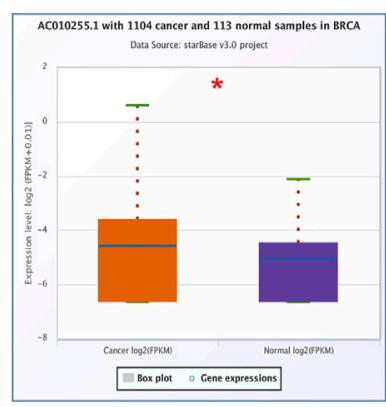

RRC75A-AS1

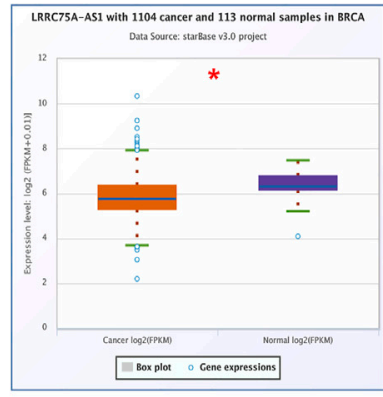

AC159540.2

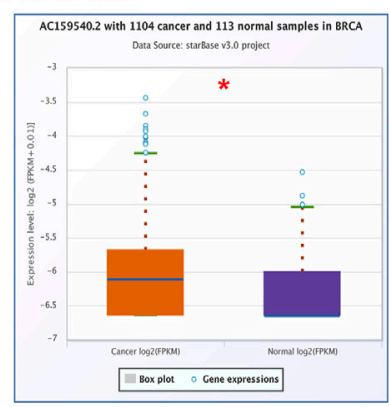

FAM239A

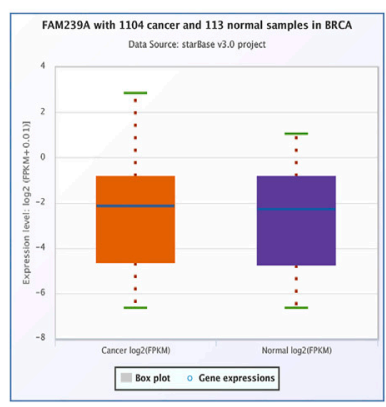

B RGPD4-AS1

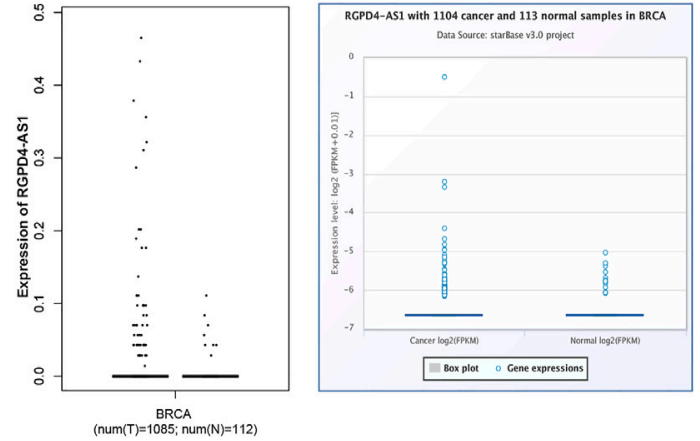

MALAT1

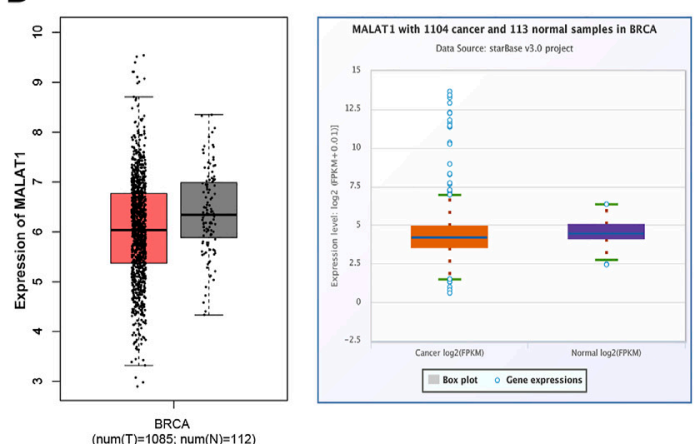

\section{F $\quad$ AC091891.2}
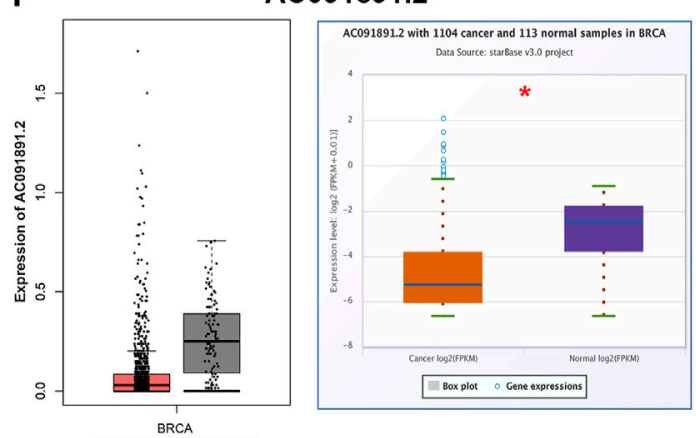

$\underset{\text { BRCA }}{\text { BRum(T) }} 1085 ;$ num $(N)=112)$

FIGURE 1 | Identification and validation of potential inflammation-related IncRNAs in breast cancer. The expression of LRRC75A-AS1 (A), RGPD4-AS1 (B), AC010255.1 (C), MALAT1 (D), AC159540.2 (E), AC091891.2 (F) and FAM239A (G) in breast cancer compared with normal controls determined by GEPIA and starBase databases. ${ }^{*} p<0.05$. 

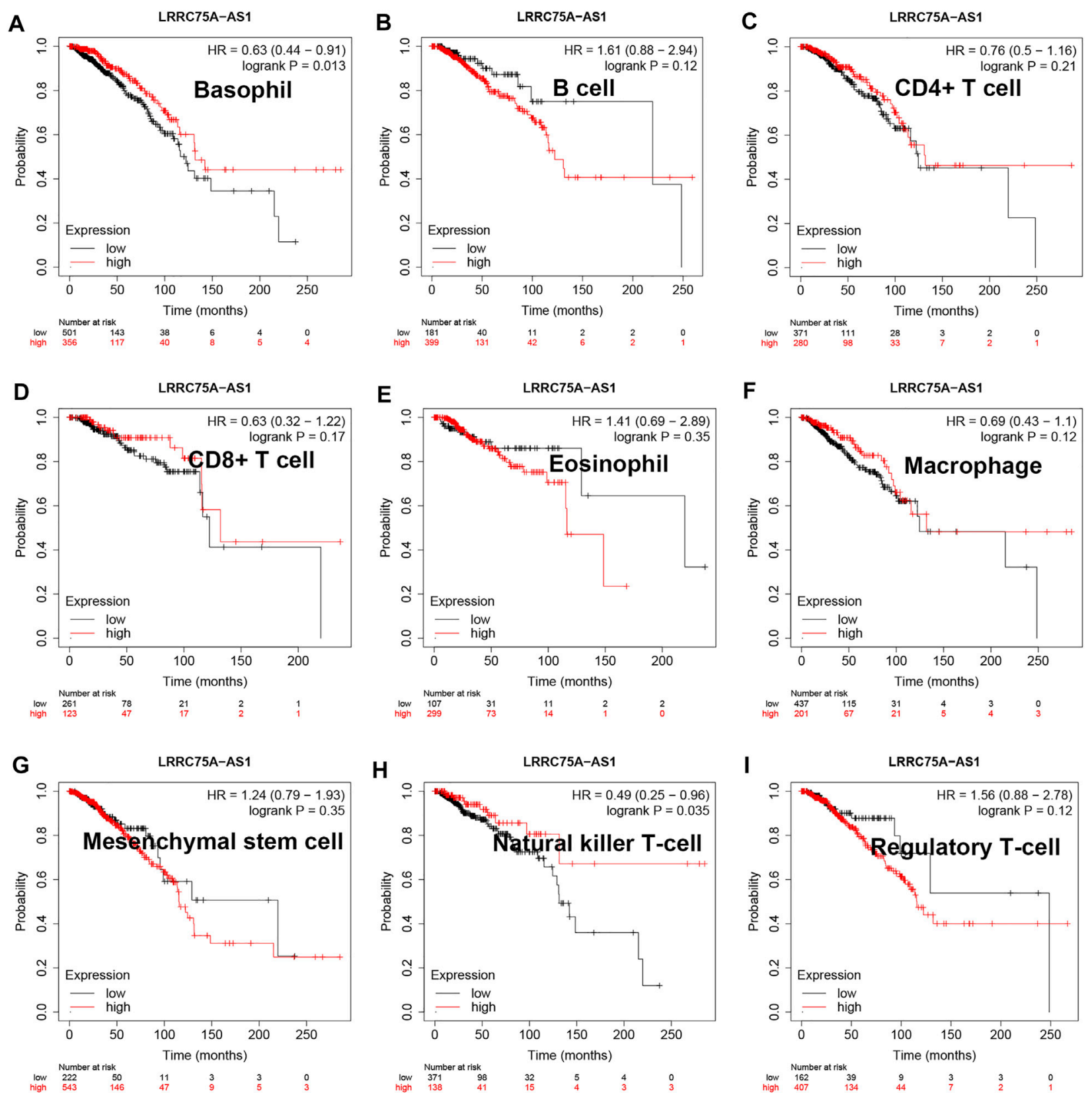

FIGURE 2 | Survival analysis for LRRC75A-AS1 in breast cancer enriched with various immunocyte. (A) The prognostic value of LRRC75A-AS1 in basophilenriched breast cancer. (B) The prognostic value of LRRC75A-AS1 in B cell-enriched breast cancer. (C) The prognostic value of LRRC75A-AS1 in CD4 ${ }^{+}$cell-enriched breast cancer. (D) The prognostic value of LRRC75A-AS1 in CD8 ${ }^{+}$cell-enriched breast cancer. (E) The prognostic value of LRRC75A-AS1 in eosinophil-enriched breast cancer. (F) The prognostic value of LRRC75A-AS1 in macrophage-enriched breast cancer. (G) The prognostic value of LRRC75A-AS1 in mesenchymal stem cellenriched breast cancer. (H) The prognostic value of LRRC75A-AS1 in natural killer T cell-enriched breast cancer. (I) The prognostic value of LRRC75A-AS1 in regulatory T cell-enriched breast cancer.

cancer using starBase database. As presented in Figure 5A, 6 miRNAs were significantly downregulated in breast cancer tissues compared with normal breast tissues, including miR149-5p, miR-3127-5p, miR-330-5p, miR-2114-3p, miR-1277$3 p$ and miR-760. The prognostic values of the overexpressed six miRNAs in breast cancer were also assessed by Kaplan-
Meier plotter database. Only breast cancer patients with high expression of miR-3127-5p or miR-2114-3p had poor prognosis. By combination of correlation analysis, expression analysis and survival analysis, miR-3127-5p and miR-2114-3p might be two most potential downstream binding miRNAs of LRRC75A-AS1 in breast cancer. 

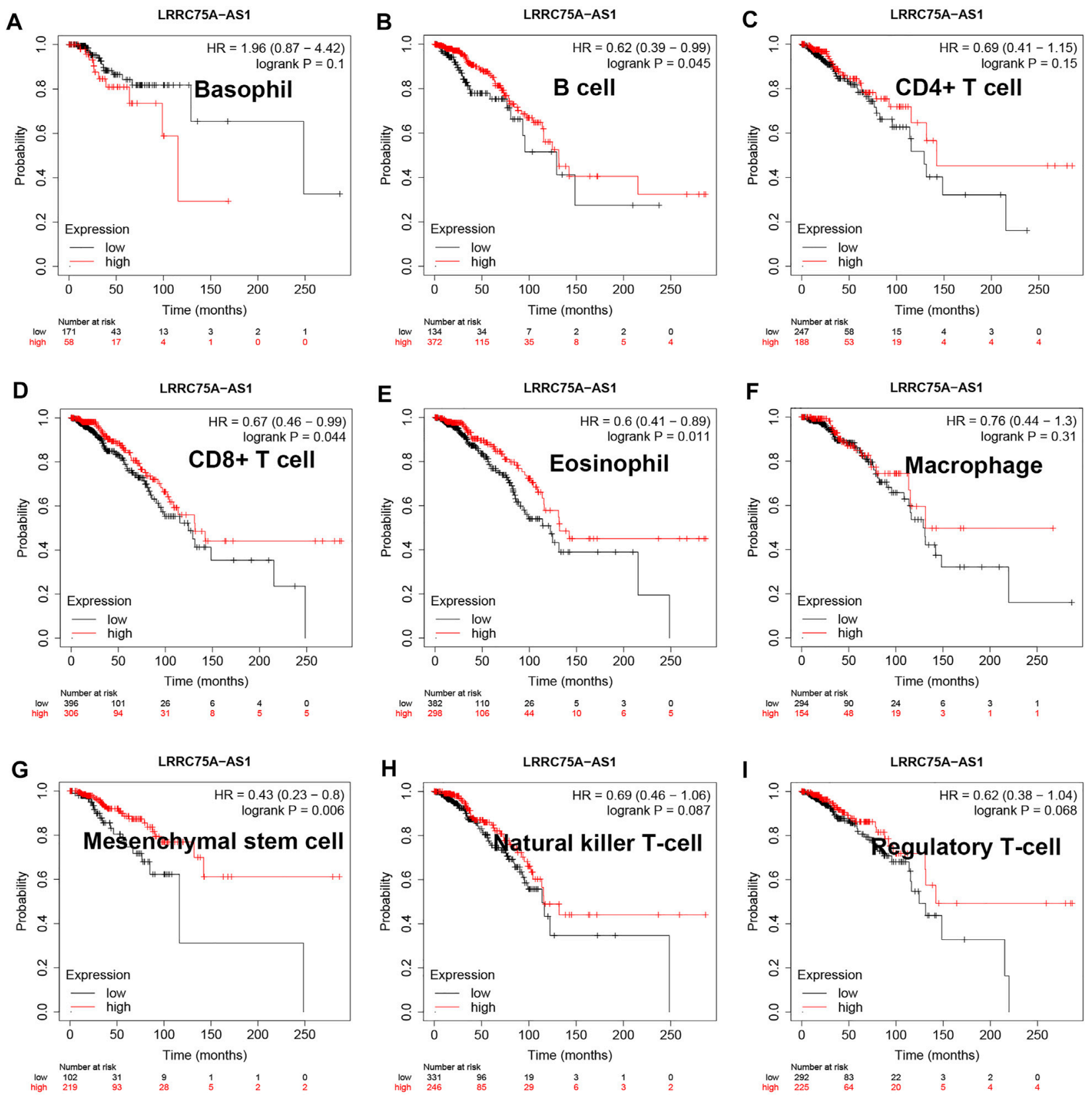

FIGURE 3 | Survival analysis for LRRC75A-AS1 in breast cancer decreased with various immunocyte. (A) The prognostic value of LRRC75A-AS1 in basophildecreased breast cancer. (B) The prognostic value of LRRC75A-AS1 in B cell-decreased breast cancer. (C) The prognostic value of LRRC75A-AS1 in CD4 ${ }^{+}$celldecreased breast cancer. (D) The prognostic value of LRRC75A-AS1 in CD8 ${ }^{+}$cell-decreased breast cancer. (E) The prognostic value of LRRC75A-AS1 in eosinophildecreased breast cancer. (F) The prognostic value of LRRC75A-AS1 in macrophage-decreased breast cancer. (G) The prognostic value of LRRC75A-AS1 in mesenchymal stem cell-decreased breast cancer. (H) The prognostic value of LRRC75A-AS1 in natural killer T cell-decreased breast cancer. (I) The prognostic value of LRRC75A-AS1 in regulatory $T$ cell-decreased breast cancer.

\section{Identification of Downstream Inflammation-Related Targets of LRRC75A-AS1-miRNA Pathways in Breast Cancer}

Next, we intended to explore the downstream molecular mechanism of inflammation-related LRRC75A-AS1-miR-3127-
$5 p / m i R-2114-3 p$ pathways in breast cancer. First of all, these genes that were significantly associated with inflammation in breast cancer were obtained by analyzing the four scRNA-seq studies as mentioned above. 2,452 inflammation-related genes were identified (Data not shown). Moreover, as is known to all, miRNAs usually exert their roles by suppressing target genes. Thus, the potential target genes of miR-3127-5p or miR-2114-3p 


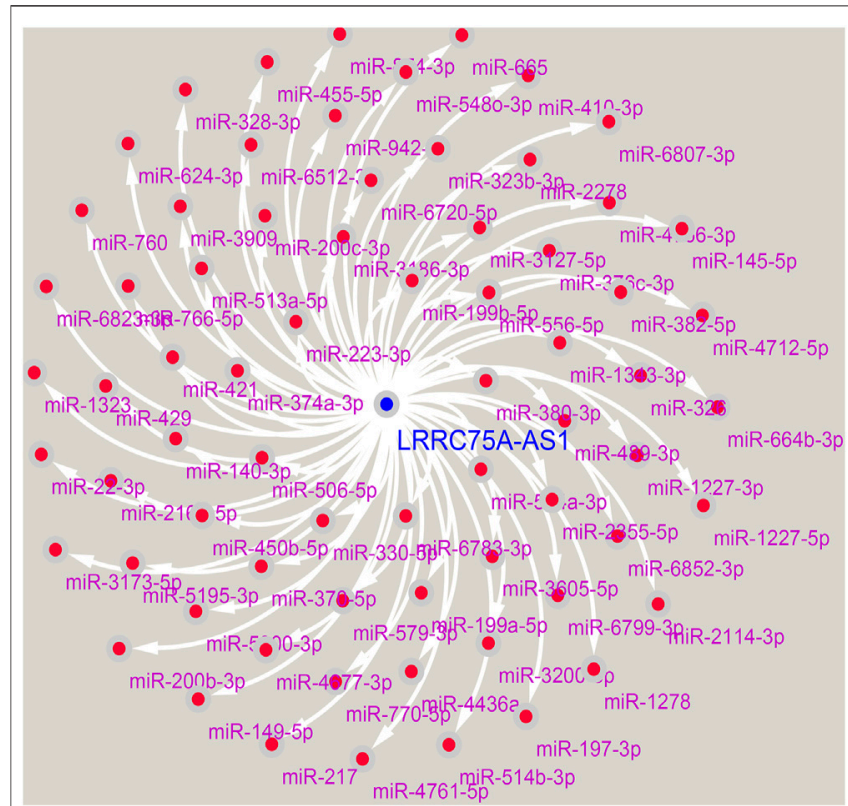

FIGURE 4 | Construction of a LRRC75A-AS1-miRNA regulatory.

were predicted by miRNet database. 64 and 75 genes were forecasted to be downstream targets of miR-3127-5p and miR2114-3p, respectively. For better visualization, miR-3127-5p-gene and miR-2114-3p-gene networks were established as shown in Figure 6A and Figure 6B. By intersection of target genes and inflammation-related genes, 11 and 9 potential inflammationrelated target genes of miR-3127-5p and miR-2114-3p were respectively identified as presented in Figures 6C,D.

\section{Construction of an Inflammation-Related LRRC75A-AS1-miRNA-mRNA Network in Breast Cancer}

Based on the negative regulation mechanism of miRNA, expression correlation analysis was conducted. As listed in Table 3, 3 of 11 target genes (DMTN, MCL1 and RPL36) were significantly inversely correlated with miR-3127-5p expression in breast cancer. And two target genes (ULK2 and RPL36) were significantly positively correlated with LRRC75AAS1 expression in breast cancer. Thus, RPL36 might be the most potential inflammation-related target gene of LRRC75A-AS1/ miR-3127-5p pathway in breast cancer. In the same light, three target genes (FABP7, RPL27A and TMCC2) were markedly negatively associated with miR-2114-3p expression in breast cancer and two target genes (RPL27A and TMCC2) were markedly positively linked to LRRC75A-AS1 expression in breast cancer as shown in Table 4. Therefore, RPL27A and TMCC2 might be two potential inflammation-related target genes of LRRC75A-AS1/miR-2114-3p pathway in breast cancer. Finally, the prognostic values of RPL36, RPL27A and TMCC2 in breast cancer were evaluated using Kaplan-Meier plotter database. As presented in Figure 7, high expression of RPL36 and RPL27A indicated favorable prognosis in breast

TABLE 2| The expression correlation of LRRC75A-AS1 with predicted miRNAs in breast cancer determined by starBase database.

IncRNA

LRRC75A-AS1

LRRC75A-AS1

LRRC75A-AS1

LRRC75A-AS1

LRRC75A-AS1

LRRC75A-AS1

LRRC75A-AS1

LRRC75A-AS1

LRRC75A-AS1

LRRC75A-AS1

LRRC75A-AS1

LRRC75A-AS1

LRRC75A-AS1

LRRC75A-AS1

LRRC75A-AS1

LRRC75A-AS1

LRRC75A-AS1

LRRC75A-AS1

LRRC75A-AS1

LRRC75A-AS1

LRRC75A-AS1

LRRC75A-AS1

LRRC75A-AS1

LRRC75A-AS1

LRRC75A-AS1

LRRC75A-AS1

LRRC75A-AS1

LRRC75A-AS1

LRRC75A-AS1

LRRC75A-AS1

LRRC75A-AS1

LRRC75A-AS1

LRRC75A-AS1

LRRC75A-AS1

LRRC75A-AS1

LRRC75A-AS1

LRRC75A-AS1

LRRC75A-AS1

LRRC75A-AS1

LRRC75A-AS1

LRRC75A-AS1

LRRC75A-AS1

LRRC75A-AS1

LRRC75A-AS1

LRRC75A-AS1

LRRC75A-AS1

LRRC75A-AS1

LRRC75A-AS1

LRRC75A-AS1

LRRC75A-AS1

LRRC75A-AS1

LRRC75A-AS1

LRRC75A-AS1

LRRC75A-AS1

LRRC75A-AS1

LRRC75A-AS1

LRRC75A-AS1

LRRC75A-AS1

LRRC75A-AS1

LRRC75A-AS1

LRRC75A-AS1

LRRC75A-AS1

LRRC75A-AS1

\begin{tabular}{|c|c|c|}
\hline miRNA & R-value & $p$-value \\
\hline miR-149-5p & -0.131 & 1.45E-05 \\
\hline miR-874-3p & -0.114 & 1.63E-04 \\
\hline miR-22-3p & -0.104 & 5.71E-04 \\
\hline miR-2355-5p & -0.101 & 8.70E-04 \\
\hline miR-3127-5p & -0.101 & 9.01E-04 \\
\hline miR-370-5p & -0.086 & 4.61E-03 \\
\hline miR-326 & -0.084 & 5.48E-03 \\
\hline miR-330-5p & -0.080 & 8.20E-03 \\
\hline miR-328-3p & -0.077 & 1.14E-02 \\
\hline miR-2114-3p & -0.071 & 1.93E-02 \\
\hline miR-1277-3p & -0.065 & 3.15E-02 \\
\hline miR-1277-5p & -0.065 & 3.28E-02 \\
\hline miR-6823-3p & -0.065 & 3.27E-02 \\
\hline miR-323b-3p & -0.063 & 3.69E-02 \\
\hline miR-760 & -0.060 & 4.99E-02 \\
\hline miR-145-5p & -0.051 & 9.63E-02 \\
\hline miR-382-5p & -0.051 & 9.16E-02 \\
\hline miR-489-3p & -0.049 & 1.06E-01 \\
\hline miR-6799-3p & -0.049 & 1.05E-01 \\
\hline miR-4766-3p & -0.047 & 1.20E-01 \\
\hline miR-2278 & -0.040 & 1.85E-01 \\
\hline miR-3909 & -0.040 & 1.88E-01 \\
\hline miR-665 & -0.039 & 2.02E-01 \\
\hline miR-4712-5p & -0.037 & 2.19E-01 \\
\hline miR-942-5p & -0.037 & 2.26E-01 \\
\hline$m i R-514 b-3 p$ & -0.033 & $2.75 \mathrm{E}-01$ \\
\hline miR-624-3p & -0.032 & 2.95E-01 \\
\hline miR-3200-5p & -0.029 & 3.40E-01 \\
\hline miR-197-3p & -0.026 & 3.94E-01 \\
\hline miR-4436a & -0.023 & 4.55E-01 \\
\hline miR-421 & -0.020 & 5.02E-01 \\
\hline miR-3173-5p & -0.017 & 5.78E-01 \\
\hline miR-766-5p & -0.016 & 5.89E-01 \\
\hline miR-548o-3p & -0.014 & 6.55E-01 \\
\hline miR-199a-5p & -0.012 & 6.87E-01 \\
\hline miR-6852-3p & -0.012 & 6.88E-01 \\
\hline miR-374a-3p & -0.011 & 7.11E-01 \\
\hline miR-579-3p & -0.011 & 7.08E-01 \\
\hline miR-1343-3p & -0.010 & 7.51E-01 \\
\hline miR-199b-5p & -0.010 & 7.37E-01 \\
\hline miR-3605-5p & -0.009 & 7.57E-01 \\
\hline miR-429 & -0.007 & 8.24E-01 \\
\hline miR-4761-5p & -0.007 & 8.16E-01 \\
\hline miR-376c-3p & -0.004 & 8.87E-01 \\
\hline miR-4677-3p & 0.002 & 9.59E-01 \\
\hline miR-664b-3p & 0.002 & 9.51E-01 \\
\hline miR-6783-3p & 0.003 & 9.26E-01 \\
\hline$m i R-450 b-5 p$ & 0.005 & 8.63E-01 \\
\hline miR-514a-3p & 0.006 & 8.46E-01 \\
\hline miR-5195-3p & 0.006 & 8.37E-01 \\
\hline miR-6807-3p & 0.012 & 6.94E-01 \\
\hline miR-5000-3p & 0.013 & 6.68E-01 \\
\hline miR-506-5p & 0.015 & 6.20E-01 \\
\hline miR-6720-5p & 0.019 & 5.35E-01 \\
\hline miR-513a-5p & 0.021 & 4.88E-01 \\
\hline$m i R-140-3 p$ & 0.032 & 2.94E-01 \\
\hline miR-200b-3p & 0.033 & 2.70E-01 \\
\hline miR-556-5p & 0.033 & 2.75E-01 \\
\hline miR-380-3p & 0.034 & 2.69E-01 \\
\hline miR-216a-5p & 0.037 & 2.23E-01 \\
\hline miR-1278 & 0.038 & 2.05E-01 \\
\hline miR-223-3p & 0.041 & 1.74E-01 \\
\hline miR-410-3p & 0.045 & 1.40E-01 \\
\hline
\end{tabular}


TABLE 2 | (Continued) The expression correlation of LRRC75A-AS1 with predicted miRNAs in breast cancer determined by starBase database.

\begin{tabular}{lccc} 
IncRNA & miRNA & R-value & $\boldsymbol{p}$-value \\
\hline LRRC75A-AS1 & miR-6512-3p & 0.047 & $1.22 \mathrm{E}-01$ \\
LRRC75A-AS1 & miR-3186-3p & 0.049 & $1.07 \mathrm{E}-01$ \\
LRRC75A-AS1 & miR-770-5p & 0.051 & $9.32 \mathrm{E}-02$ \\
LRRC75A-AS1 & miR-1323 & 0.068 & $2.43 \mathrm{E}-02$ \\
LRRC75A-AS1 & miR-200c-3p & 0.073 & $1.59 \mathrm{E}-02$ \\
LRRC75A-AS1 & miR-217 & 0.074 & $1.43 \mathrm{E}-02$ \\
LRRC75A-AS1 & $m i R-455-5 p$ & 0.127 & $2.59 E-05$
\end{tabular}

cancer. No statistical role of TMCC2 in predicting prognosis of breast cancer was observed. Taken together, an inflammationrelated LRRC75A-AS1-mediated miRNA-mRNA pathways in breast cancer were identified as vividly depicted in Figure 8.

\section{DISCUSSION}

Despite inflammation is present in breast cancer and may affect its outcome, the molecular mechanism of inflammation in breast

\section{A}

\begin{tabular}{lccccccccc|} 
miRNA & miR-149-5p & miR-874-3p & miR-22-3p & miR-2355-5p & miR-3127-5p & miR-370-5p & miR-326 & miR-330-5p \\
FC & 2.22 & 0.82 & 0.67 & 0.89 & 2.28 & 0.41 & 0.42 & 1.67 \\
P-value & $4.50 \mathrm{E}-02$ & $1.40 \mathrm{E}-07$ & $1.10 \mathrm{E}-15$ & $2.60 \mathrm{E}-03$ & $4.00 \mathrm{E}-15$ & $9.80 \mathrm{E}-18$ & $9.30 \mathrm{E}-17$ & $4.30 \mathrm{E}-04$ \\
miRNA & miR-328-3p & miR-2114-3 & miR-1277-3 & miR-1277-5p & miR-6823-3p miR-323b-3p & miR-760 & \\
FC & 0.55 & 7.77 & 3.84 & 1.34 & 0.87 & 1.30 & 6.43 \\
P-value & $2.40 \mathrm{E}-19$ & $8.90 \mathrm{E}-09$ & $9.50 \mathrm{E}-24$ & $2.30 \mathrm{E}-01$ & $3.80 \mathrm{E}-01$ & $5.90 \mathrm{E}-02$ & $9.30 \mathrm{E}-20$
\end{tabular}

B

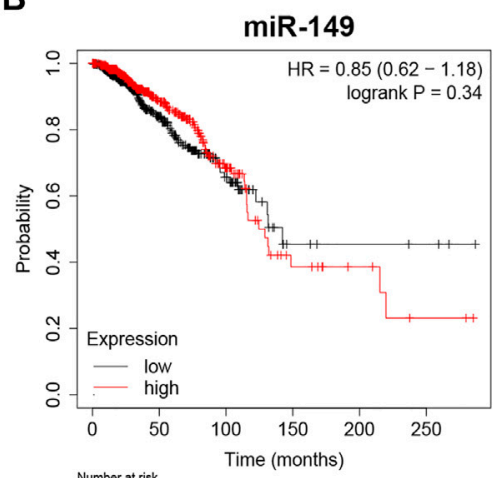

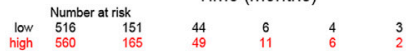

E

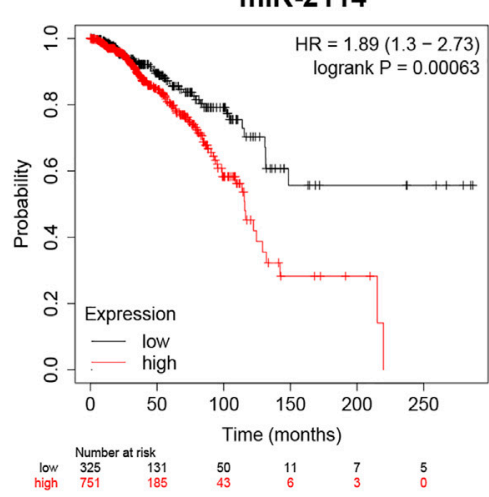

C

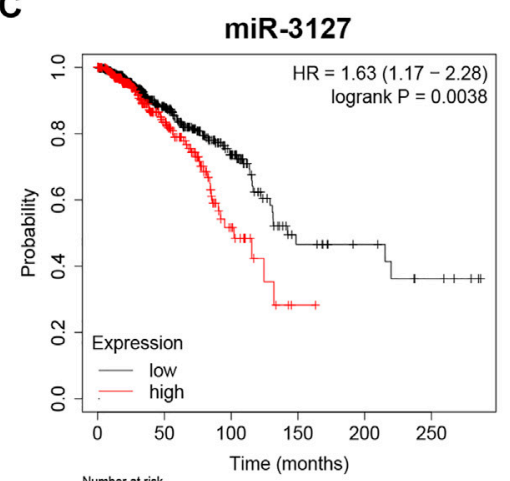

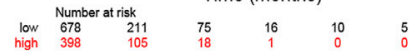

$\mathbf{F}$

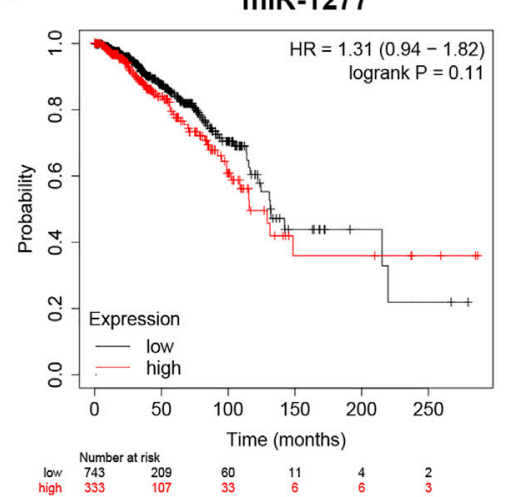

D

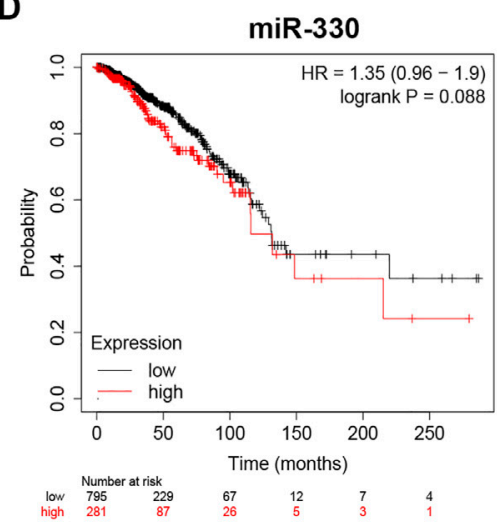

FIGURE 5 | Expression analysis and survival analysis for the miRNAs negatively correlated with LRRC75A-AS1 in breast cancer. (A) The expression levels of 15 LRRC75A-AS1-related miRNAs in breast cancer detected by starBase database. Red: overexpressed in breast cancer; Green: downregulated in breast cancer; gray: no significant differences between breast cancer and normal controls. (B-G) The prognostic value of miR-149-5p (B), miR-3127-5p (C), miR-330-5p (D), miR-2114-3p (E) and miR-1277-3p (F) and miR-760 (G) in breast cancer. 

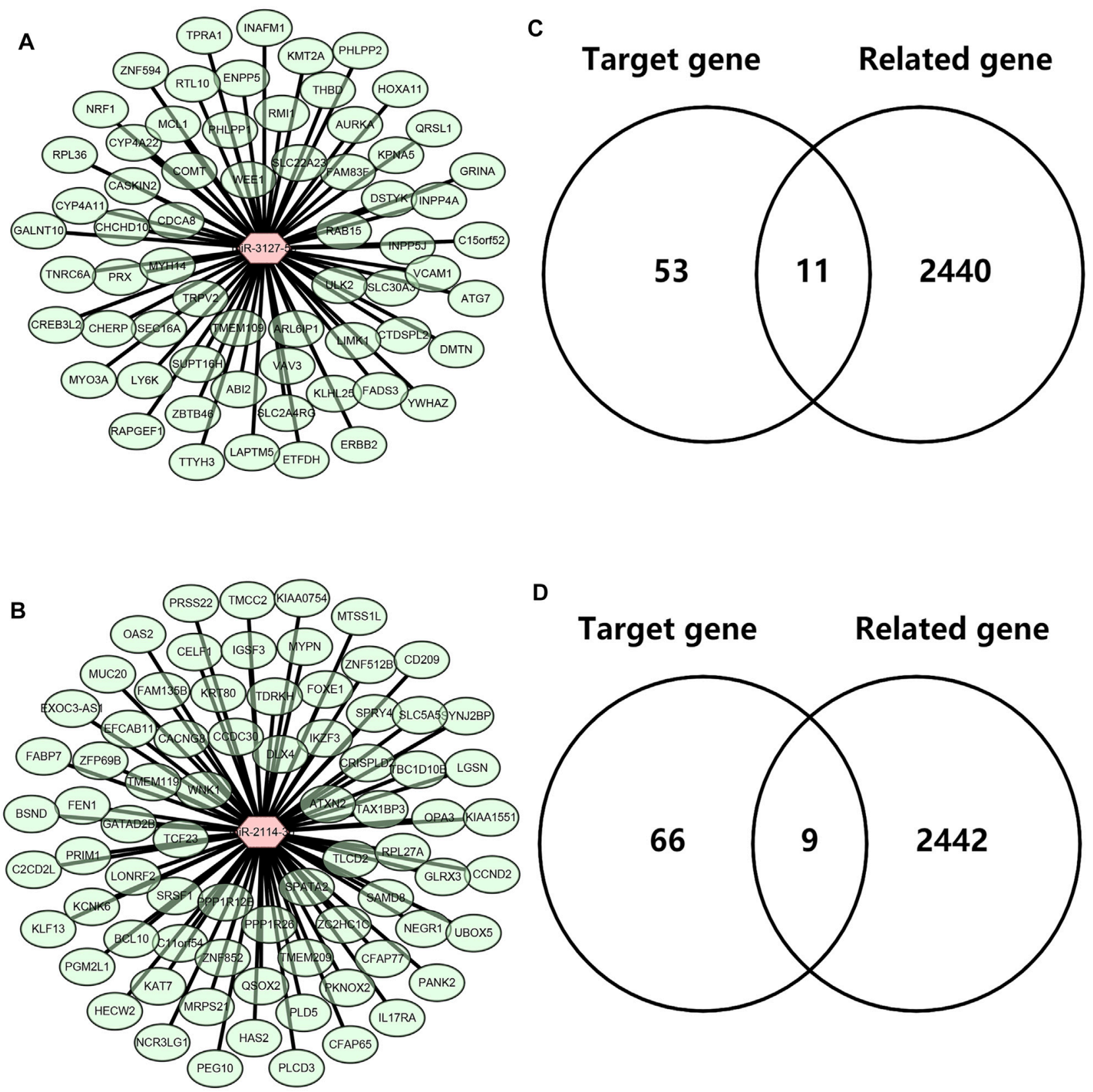

D

\section{Target gene Related gene}

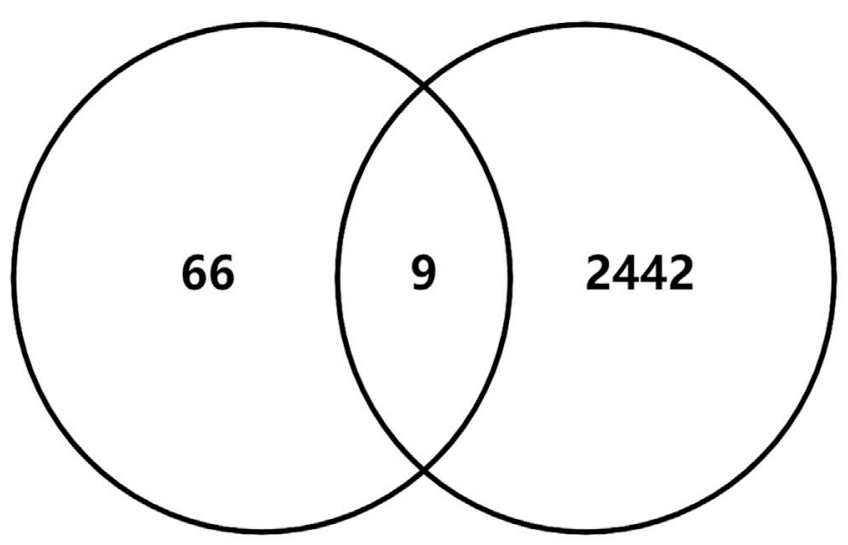

FIGURE 6 | Identification of downstream potential targets of miR-3127-5p and miR-2114-3p in breast cancer. (A) Establishment of a miR-3127-5p-gene network. (B) Establishment of a miR-2114-3p-gene network. (C,D) Intersection of predicted target genes of miR-3127-5p (C) or miR-2114-3p (D) and inflammation-related genes in breast cancer.

carcinogenesis is still unknown. In this study, we aimed to identify a lncRNA-miRNA-mRNA regulatory network based on ceRNA hypothesis at single-cell level.

CancerSEA was employed to identify inflammation-related lncRNAs in breast cancer at single-cell resolution. By integrated analysis of lncRNAs' expression, LRRC75A-AS1 was identified as the most potential inflammation-associated lncRNA in breast cancer. Several studies have confirmed its key roles in human cancer. For example, Pang et al. suggested that LRRC75A-AS1 suppressed multiple myeloma by targeting miR-199b-5p/PDCD4 axis (Pang et al., 2020); Chen et al. demonstrated that LRRC75AAS1 repressed cell proliferation and migration of colorectal carcinoma (Chen et al., 2019); Li et al. confirmed that LRRC75A-AS1 facilitated cell proliferation and invasion of triple negative breast cancer by acting as a ceRNA to regulate BAALC (Li et al., 2020). Tumor infiltrating lymphocytes (TILs) are closely linked to prognosis of cancer patients (Yu S et al., 2020; Lou et al., 2021). Moreover, survival analysis also revealed that 
TABLE 3 | The expression correlation of miR-3127-5p with target genes or target genes with LRRC75A-AS1 in breast cancer.

\begin{tabular}{lccccc}
\hline \multirow{2}{*}{ Target gene } & \multicolumn{2}{c}{$\mathbf{m i R - 3 1 2 7 - 5 p}$} & & \multicolumn{2}{c}{ LRRC75A-AS1 } \\
\cline { 2 - 3 } & R-value & $\boldsymbol{p}$-value & & R-value & $\boldsymbol{p}$-value \\
\hline DMTN & -0.148 & $1.02 \mathrm{E}-06$ & & 0.062 & $3.81 \mathrm{E}-02$ \\
ERBB2 & 0.045 & $1.41 \mathrm{E}-01$ & & -0.190 & $2.08 \mathrm{E}-10$ \\
ETFDH & -0.040 & $1.85 \mathrm{E}-01$ & & 0.004 & $9.00 \mathrm{E}-01$ \\
GRINA & 0.230 & $1.74 \mathrm{E}-14$ & & -0.074 & $1.36 \mathrm{E}-02$ \\
MCL1 & -0.182 & $1.65 \mathrm{E}-09$ & & 0.034 & $2.59 \mathrm{E}-01$ \\
LAPTM5 & -0.070 & $2.10 \mathrm{E}-02$ & & -0.113 & $1.68 \mathrm{E}-04$ \\
ULK2 & 0.044 & $1.50 \mathrm{E}-01$ & & 0.180 & $1.83 \mathrm{E}-09$ \\
VAV3 & 0.008 & $7.82 \mathrm{E}-01$ & & -0.087 & $3.93 \mathrm{E}-03$ \\
SUPT16H & 0.273 & $6.13 \mathrm{E}-20$ & & -0.011 & $7.08 \mathrm{E}-01$ \\
RPL36 & $-\mathbf{0 . 1 5 1}$ & $\mathbf{6 . 3 4 E}-\mathbf{0 7}$ & $\mathbf{0 . 4 3 5}$ & $\mathbf{2 . 7 8 E - 5 2}$ \\
QRSL1 & 0.079 & $9.04 \mathrm{E}-03$ & & 0.020 & $5.13 \mathrm{E}-01$
\end{tabular}

LRRC75A-AS1 possessed significant prognostic value in breast cancer enriched with basophil, natural killer T cell, or decreased with B cell, $\mathrm{CD}^{+} \mathrm{T}$ cell, eosinophil or mesenchymal stem cell.

ceRNA mechanism can partially account for lncRNA's action mechanism (Lou et al., 2020). Using miRNet database, a series of miRNAs were predicted. By combination of correlation analysis, expression analysis and survival analysis, miR-3127-5p and miR2114-3p were selected as the two most potential downstream binding miRNAs of LRRC75A-AS1 in breast cancer. Both of the two miRNAs have been reported to be involved in initiation and progression of human malignancies, including osteosarcoma (Wang et al., 2021), papillary thyroid cancer (Zhou et al., 2021), colorectal cancer (Ma et al., 2021) and ovarian cancer (Yu Z et al., 2020).

Subsequently, the downstream target genes of miR-3127-5p or miR-2114-3p were predicted by online target gene prediction tool. Moreover, the inflammation-related genes in breast cancer were also identified using four breast cancer scRNA-seq studies as mentioned above. By intersection of target gene set and inflammation-related gene sets, 11 and 9 candidate genes were screened out for miR-3127-5p and miR-2114-3p, respectively. After performing correlation analysis and survival analysis, RPL36 was identified as the most potential target for miR3127-5p and RPL27A was for miR-2114-3p.

RPL36 was reported as a tumor suppressor to restrain KRASinduced pancreatic cancer (Provost et al., 2014), and was a promising prognostic marker in hepatocellular carcinoma (Song et al., 2011). RPL27A was also found to be involved in development and metastasis of triple-negative breast cancer in both mouse and human (Zhao et al., 2021). Our analytic results together with these reports indicated that RPL36 and RPL27A might be downstream key targets of LRRC75A-AS1/miR-3127$5 \mathrm{p} / \mathrm{miR}-2114-3 \mathrm{p}$ pathways in breast cancer.

At the end, a novel inflammation-related lncRNA-miRNAmRNA regulatory network in breast cancer was successively established based on ceRNA hypothesis and scRNA-seq data. There were several limitations in this study. For example, the miRNAs in this network were not identified using scRNA-seq data; the results from this study were only based on in silico analysis. However, this is the first study to comprehensively explore inflammation-related lncRNA-miRNA-mRNA network in breast cancer. All these findings need to be further validated using basic experiments and clinical trials in the future.

\section{MATERIALS AND METHODS}

\section{Screening of Inflammation-Related Long Non-Coding RNAs and Genes}

CancerSEA (http://biocc.hrbmu.edu.cn/CancerSEA/home.jsp) is the first dedicated database that aims to comprehensively decode distinct functional states of cancer cells at single-cell resolution (Yuan et al., 2019). In this study, CancerSEA database was employed to identify inflammation-related lncRNAs and genes in breast cancer. Only those lncRNAs and genes that were significantly associated with inflammation in breast cancer were included. $p$-value $<0.05$ was considered as statistically significant.

\section{Expression Analysis for Long Non-Coding RNA}

Two databases, including GEPIA (http://gepia.cancer-pku.cn/) and starBase (http://starbase.sysu.edu.cn/), were used to analyze the expression of lncRNAs in breast cancer (Li et al., 2014; Tang et al., 2017). Only lncRNAs that were significantly upregulated or downregulated in both two databases were selected for subsequent analysis. $p$-value $<0.05$ was considered as statistically significant.

\section{Survival Analysis for Long Non-Coding RNA}

The prognostic values of LRRC75A-AS1 in breast cancer were determined using Kaplan-Meier plotter (http://kmplot.com/ analysis/), which is an online database capable of accessing the effects of genes or miRNAs on survival in more than 20 cancer types including breast cancer (Lou et al., 2021). Logrank $p$-value < 0.05 was considered as statistically significant.

\section{MicroRNA Prediction}

miRNet (http://www.mirnet.ca), a database for miRNA functional analysis and systems biology, was used to predict the binding miRNAs of LRRC75A-AS1 (Chang et al., 2020).

TABLE 4 | The expression correlation of miR-2114-3p with target genes or target genes with LRRC75A-AS1 in breast cancer.

\begin{tabular}{|c|c|c|c|c|}
\hline \multirow[t]{2}{*}{ Target gene } & \multicolumn{2}{|c|}{ miR-2114-3p } & \multicolumn{2}{|c|}{ LRRC75A-AS1 } \\
\hline & R-value & $p$-value & R-value & $p$-value \\
\hline FABP7 & -0.125 & 3.82E-05 & 0.056 & 6.31E-02 \\
\hline RPL27A & -0.084 & 5.77E-03 & 0.472 & 2.61E-62 \\
\hline TMCC2 & -0.098 & $1.25 E-03$ & 0.108 & $3.45 E-04$ \\
\hline KAT7 & 0.109 & $3.41 \mathrm{E}-04$ & -0.104 & 5.23E-04 \\
\hline SYNJ2BP & 0.104 & 5.90E-04 & -0.165 & 3.85E-08 \\
\hline TMEM209 & 0.030 & 3.31E-01 & -0.043 & 1.52E-01 \\
\hline EFCAB11 & 0.167 & $3.15 \mathrm{E}-08$ & -0.058 & 5.39E-02 \\
\hline NEGR1 & 0.061 & 4.59E-02 & -0.004 & 8.90E-01 \\
\hline TMEM119 & 0.039 & 2.03E-01 & -0.049 & 1.02E-01 \\
\hline
\end{tabular}



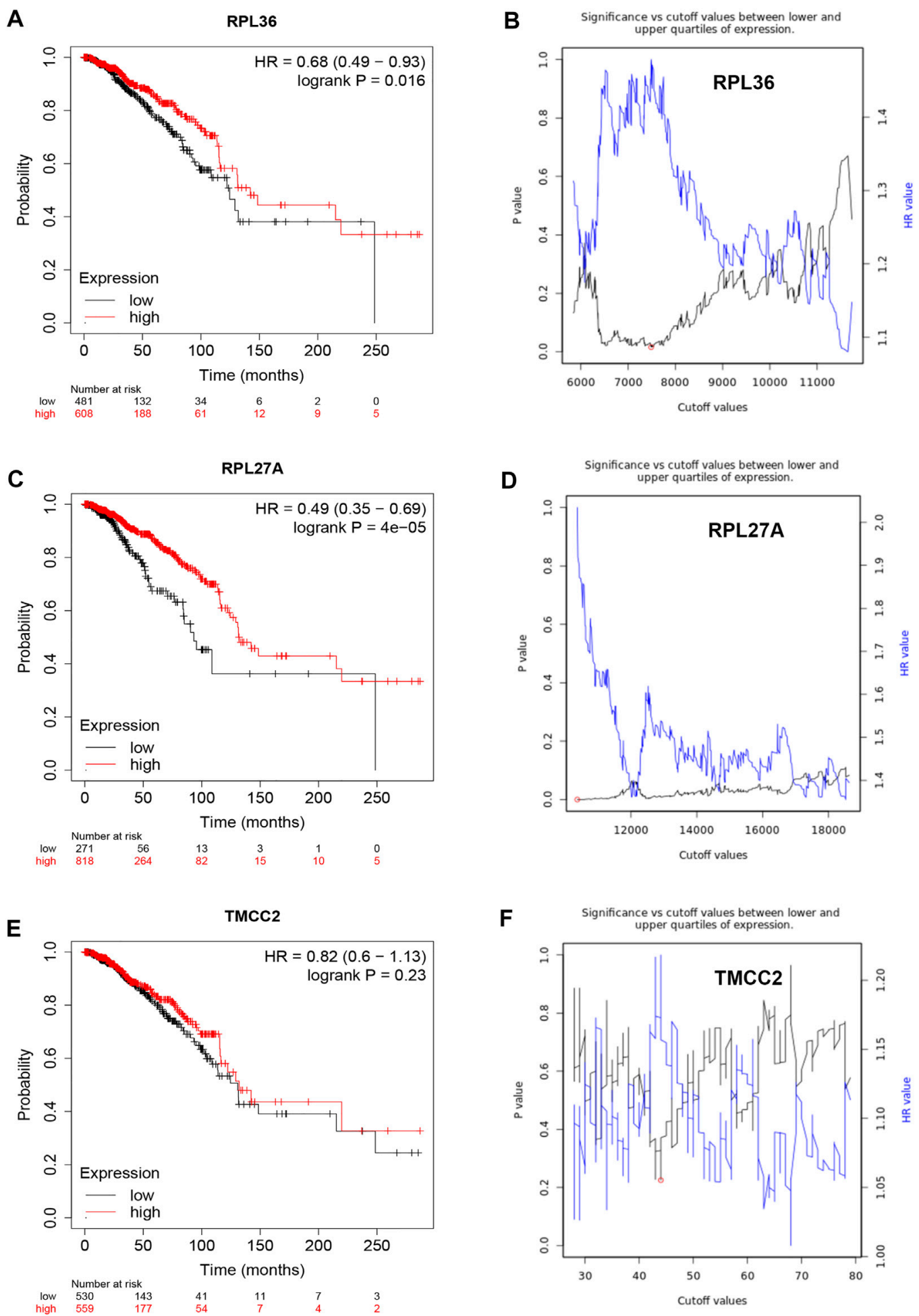

FIGURE 7 | Survival analysis for RPL36, RPL27A and TMCC2 in breast cancer. (A) The prognostic value of RPL36 in breast cancer. (B) The plot of significance versus cutoff values between lower and upper quartiles of RPL36 expression. (C) The prognostic value of RPL27A in breast cancer. (D) The plot of significance versus cutoff values between lower and upper quartiles of RPL27A expression. (E) The prognostic value of TMCC2 in breast cancer. (F) The plot of significance versus cutoff values between lower and upper quartiles of TMCC2 expression. 


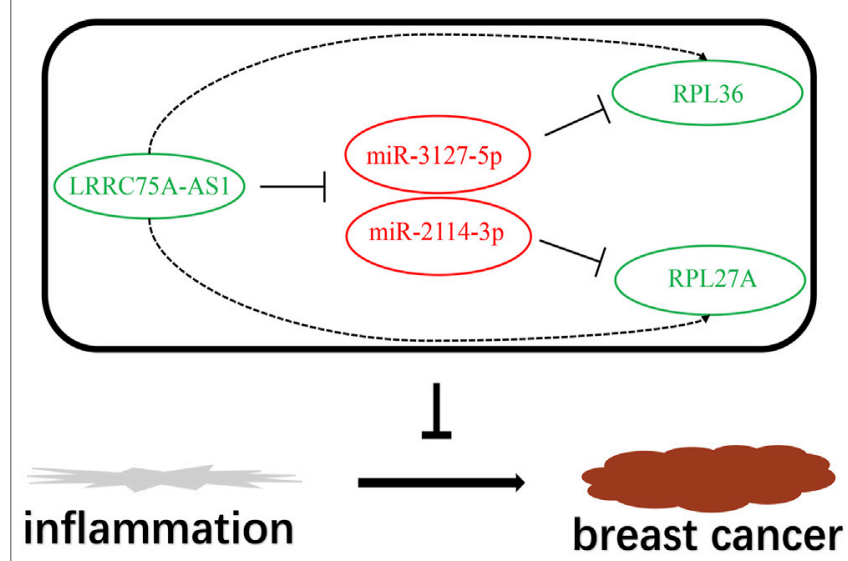

FIGURE 8 | The model of LRRC75A-AS1-mediated inflammationrelated miRNA-mRNA pathways in breast cancer.

Then, a LRRC75A-AS1-miRNA network was established using Cytoscape software.

\section{Expression and Survival Analysis for MicroRNA}

The expression levels of miRNAs of LRRC75A-AS1 in breast cancer were determined by usage of starBase (http://starbase.sysu. edu.cn/). $p$-value $<0.05$ was considered as statistically significant. Kaplan-Meier plotter (http://kmplot.com/analysis/) was employed to assess the prognostic values of miRNAs of LRRC75A-AS1. The survival plots were downloaded from the website. Logrank $p$-value $<0.05$ was considered as statistically significant.

\section{Target Gene Prediction}

miRNet (http://www.mirnet.ca) was introduced to predict the downstream target genes of miR-3127-5p and miR-2114-3p. Then, a miR-3127-5p-target gene network or miR-2114-3ptarget gene network was constructed using Cytoscape software.

\section{Intersection Analysis}

VENNY 2.1 (https://bioinfogp.cnb.csic.es/tools/venny/index. html) was employed to perform intersection analysis for target genes of miRNAs and inflammation-related genes in breast

\section{REFERENCES}

Balavenkatraman, K. K., Aceto, N., Britschgi, A., Mueller, U., Bence, K. K., Neel, B. G., et al. (2011). Epithelial Protein-Tyrosine Phosphatase 1B Contributes to the Induction of Mammary Tumors by HER2/Neu but Is Not Essential for Tumor Maintenance. Mol. Cancer Res. 9, 1377-1384. doi:10.1158/1541-7786.mcr-11-0198

Balkwill, F., and Mantovani, A. (2001). Inflammation and Cancer: Back to Virchow? Lancet 357, 539-545. doi:10.1016/s0140-6736(00)04046-0

Braune, E.-B., Tsoi, Y. L., Phoon, Y. P., Landor, S., Silva Cascales, H., Ramsköld, D., et al. (2016). Loss of CSL Unlocks a Hypoxic Response and Enhanced Tumor Growth Potential in Breast Cancer Cells. Stem Cel Rep. 6, 643-651. doi:10.1016/ j.stemcr.2016.03.004 cancer. Only the genes that commonly appeared in both two gene sets were included for subsequent analysis.

\section{Correlation Analysis}

starBase (http://starbase.sysu.edu.cn/) was used to conduct expression correlation analysis for LRRC75A-AS1-miRNA, miRNA-target gene or LRRC75A-AS1-target gene pairs in breast cancer. Only those RNA-RNA pairs with $p$-value less than 0.05 were considered as statistically significant.

\section{Statistical Analysis}

The statistical analyses were directly performed using online databases or tools as mentioned above. $p$-value $<0.05$ or logrank $p$-value $<0.05$ was considered as statistically significant.

\section{DATA AVAILABILITY STATEMENT}

The original contributions presented in the study are included in the article/Supplementary Material, further inquiries can be directed to the corresponding author.

\section{AUTHOR CONTRIBUTIONS}

JC: designed this work; JL: performed in silico analyses and wrote this manuscript. SX: performed some in silico analyses. JC: revised the manuscript. The authors have read and approved the final version of the manuscript.

\section{FUNDING}

This project was supported from the Scientific Research Fund of Zhejiang Provincial Education Department (Y202043573) and 2019 Jiaxing Key Discipiline of Medicine-Oncology (Supporting subject, No. 2019-ZC-11).

\section{SUPPLEMENTARY MATERIAL}

The Supplementary Material for this article can be found online at: https://www.frontiersin.org/articles/10.3389/fcell.2022.839876/ full\#supplementary-material

Chang, L., Zhou, G., Soufan, O., and Xia, J. (2020). miRNet 2.0: Network-Based Visual Analytics for miRNA Functional Analysis and Systems Biology. Nucleic Acids Res. 48, W244-w251. doi:10.1093/nar/gkaa467

Chen, J., Lan, J., Ye, Z., Duan, S., Hu, Y., Zou, Y., et al. (2019). Long Noncoding RNA LRRC75A-AS1 Inhibits Cell Proliferation and Migration in Colorectal Carcinoma. Exp. Biol. Med. (Maywood) 244, 1137-1143. doi:10.1177/ 1535370219874339

Chung, W., Eum, H. H., Lee, H.-O., Lee, K.-M., Lee, H.-B., Kim, K.-T., et al. (2017). Single-cell RNA-Seq Enables Comprehensive Tumour and Immune Cell Profiling in Primary Breast Cancer. Nat. Commun. 8, 15081. doi:10.1038/ ncomms 15081

Danforth, D. N. (2021). The Role of Chronic Inflammation in the Development of Breast Cancer. Cancers (Basel) 13, 3918. doi:10.3390/cancers13153918 
de la Mare, J.-A., Contu, L., Hunter, M., Moyo, B., Sterrenberg, J., Dhanani, K., et al. (2014). Breast Cancer: Current Developments in Molecular Approaches to Diagnosis and Treatment. Recent Pat Anticancer Drug Discov. 9, 153-175. doi:10.2174/15748928113086660046

Jordan, N. V., Bardia, A., Wittner, B. S., Benes, C., Ligorio, M., Zheng, Y., et al. (2016). HER2 Expression Identifies Dynamic Functional States within Circulating Breast Cancer Cells. Nature 537, 102-106. doi:10.1038/nature19328

Li, J.-H., Liu, S., Zhou, H., Qu, L.-H., and Yang, J.-H. (2014). starBase v2.0: Decoding miRNA-ceRNA, miRNA-ncRNA and Protein-RNA Interaction Networks from Large-Scale CLIP-Seq Data. Nucl. Acids Res. 42, D92-D97. doi:10.1093/nar/gkt1248

Li, S., Wu, D., Jia, H., and Zhang, Z. (2020). Long Non-coding RNA LRRC75A-AS1 Facilitates Triple Negative Breast Cancer Cell Proliferation and Invasion via Functioning as a ceRNA to Modulate BAALC. Cell Death Dis. 11, 643. doi:10. 1038/s41419-020-02821-2

Lou, W., Ding, B., and Fu, P. (2020). Pseudogene-Derived lncRNAs and Their miRNA Sponging Mechanism in Human Cancer. Front. Cel Dev. Biol. 8, 85. doi: $10.3389 /$ fcell.2020.00085

Lou, W., Wang, W., Chen, J., Wang, S., and Huang, Y. (2021). ncRNAs-mediated High Expression of SEMA3F Correlates with Poor Prognosis and Tumor Immune Infiltration of Hepatocellular Carcinoma. Mol. Ther. Nucleic Acids 24, 845-855. doi:10.1016/j.omtn.2021.03.014

Ma, Y., Li, Y., Tang, Y., Tang, N., Wang, D., and Li, X. (2021). LINC00174 Facilitates Proliferation and Migration of Colorectal Cancer Cells via MiR3127-5p/E2F7 Axis. J. Microbiol. Biotechnol. 31, 1098-1108. doi:10.4014/jmb. 2103.03001

Multhoff, G., Molls, M., and Radons, J. (2011). Chronic Inflammation in Cancer Development. Front. Immunol. 2, 98. doi:10.3389/fimmu.2011.00098

Pang, Q., Wang, Y., Bi, D., and Lu, H. (2020). LRRC75A-AS1 Targets miR-199b5p/PDCD4 axis to Repress Multiple Myeloma. Cancer Biol. Ther. 21, 1051-1059. doi:10.1080/15384047.2020.1831373

Provost, E., Bailey, J. M., Aldrugh, S., Liu, S., Iacobuzio-Donahue, C., and Leach, S. D. (2014). The Tumor Suppressor Rpl36 Restrains KRASG12V-Induced Pancreatic Cancer. Zebrafish 11, 551-559. doi:10.1089/zeb.2014.1024

Ren, L., Li, J., Wang, C., Lou, Z., Gao, S., Zhao, L., et al. (2021). Single Cell RNA Sequencing for Breast Cancer: Present and Future. Cell Death Discov. 7, 104. doi:10.1038/s41420-021-00485-1

Salmena, L., Poliseno, L., Tay, Y., Kats, L., and Pandolfi, P. P. (2011). A ceRNA Hypothesis: the Rosetta Stone of a Hidden RNA Language? Cell 146, 353-358. doi:10.1016/j.cell.2011.07.014

Song, M. J., Jung, C. K., Park, C.-H., Hur, W., Choi, J. E., Bae, S. H., et al. (2011). RPL36 as a Prognostic Marker in Hepatocellular Carcinoma. Pathol. Int. 61, 638-644. doi:10.1111/j.1440-1827.2011.02716.x

Sung, H., Ferlay, J., Siegel, R. L., Laversanne, M., Soerjomataram, I., Jemal, A., et al. (2021). Global Cancer Statistics 2020: GLOBOCAN Estimates of Incidence and
Mortality Worldwide for 36 Cancers in 185 Countries. CA A. Cancer J. Clin. 71, 209-249. doi:10.3322/caac.21660

Tang, Z., Li, C., Kang, B., Gao, G., Li, C., and Zhang, Z. (2017). GEPIA: a Web Server for Cancer and normal Gene Expression Profiling and Interactive Analyses. Nucleic Acids Res. 45, W98-w102. doi:10.1093/nar/gkx247

Wang, X., Yu, X., Long, X., and Pu, Q. (2021). MIR205 Host Gene (MIR205HG) Drives Osteosarcoma Metastasis via Regulating the microRNA 2114-3p (miR2114-3p)/twist Family bHLH Transcription Factor 2 (TWIST2) axis. Bioengineered 12, 1576-1586. doi:10.1080/21655979.2021.1920326

Yu S, S., Hu, C., Cai, L., Du, X., Lin, F., Yu, Q., et al. (2020). Seven-Gene Signature Based on Glycolysis Is Closely Related to the Prognosis and Tumor Immune Infiltration of Patients with Gastric Cancer. Front. Oncol. 10, 1778. doi:10.3389/ fonc.2020.01778

Yu Z, Z., Wang, Y., Wang, B., and Zhai, J. (2020). Metformin Affects Paclitaxel Sensitivity of Ovarian Cancer Cells through Autophagy Mediated by Long Noncoding RNASNHG7/miR-3127-5p Axis. Cancer Biother. Radiopharm. doi:10.1089/cbr.2019.3390

Yuan, H., Yan, M., Zhang, G., Liu, W., Deng, C., Liao, G., et al. (2019). CancerSEA: a Cancer Single-Cell State Atlas. Nucleic Acids Res. 47, D900-d908. doi:10.1093/ nar/gky939

Zhao, W., Li, X., Nian, W., Wang, J., Wang, X., Sun, L., et al. (2021). Ribosome Proteins Represented by RPL27A Mark the Development and Metastasis of Triple-Negative Breast Cancer in Mouse and Human. Front. Cel Dev. Biol. 9, 716730. doi:10.3389/fcell.2021.716730

Zhou, B., Ge, Y., Shao, Q., Yang, L., Chen, X., and Jiang, G. (2021). Long Noncoding RNA LINC00284 Facilitates Cell Proliferation in Papillary Thyroid Cancer via Impairing miR-3127-5p Targeted E2F7 Suppression. Cel Death Discov. 7, 156. doi:10.1038/s41420-021-00551-8

Conflict of Interest: The authors declare that the research was conducted in the absence of any commercial or financial relationships that could be construed as a potential conflict of interest.

Publisher's Note: All claims expressed in this article are solely those of the authors and do not necessarily represent those of their affiliated organizations, or those of the publisher, the editors and the reviewers. Any product that may be evaluated in this article, or claim that may be made by its manufacturer, is not guaranteed or endorsed by the publisher.

Copyright (c) 2022 Liu, Xiao and Chen. This is an open-access article distributed under the terms of the Creative Commons Attribution License (CC BY). The use, distribution or reproduction in other forums is permitted, provided the original author(s) and the copyright owner(s) are credited and that the original publication in this journal is cited, in accordance with accepted academic practice. No use, distribution or reproduction is permitted which does not comply with these terms. 